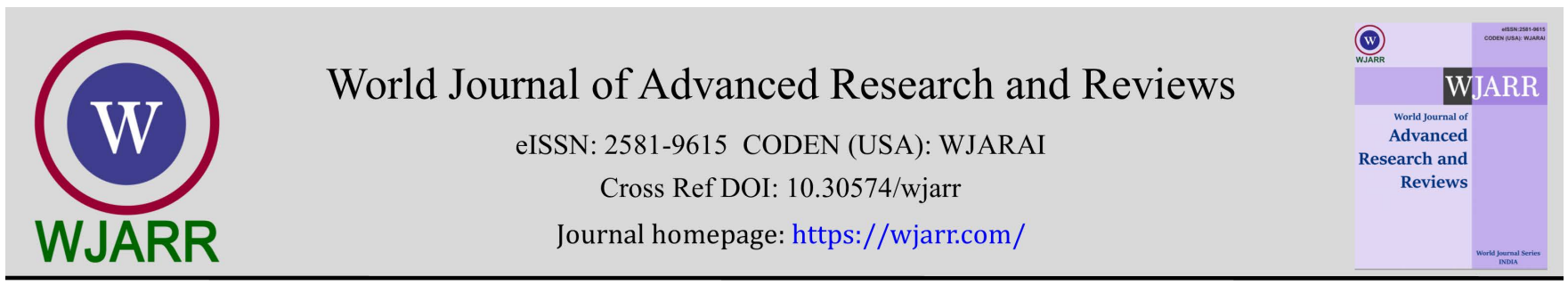

(REVIEW ARTICLE)

\title{
Cesarean sections in Greece. How can we stop the vicious cycle?
}

\author{
Evangelia Antoniou *, Eirini Orovou and Maria Iliadou \\ Department of Midwifery, University of West Attica, Greece.
}

World Journal of Advanced Research and Reviews, 2021, 12[01], 375-377

Publication history: Received on 10 September 2021; revised on 21 October 2021; accepted on 23 October 2021

Article DOI: https://doi.org/10.30574/wjarr.2021.12.1.0502

\begin{abstract}
Cesarean section has a long history in the evolution of obstetrics and human culture. Once, while a cesarean section was performed on a dying woman in order to save the infant's life, today its rates in high and middle income countries have increased dramatically. However, the increased rates of cesarean sections are not justified by the WHO recommendations in 2015 which stated that CS rates above 10-15\% were not associated with lower maternal or neonatal mortality. Greece belongs to the countries with very high rates of cesarean sections. As a result, about 6 in 10 Greek women give birth by caesarean section. For this situation, many driving factors of cesarean sections and potential solutions have been discussed. However, despite the concerns of health care professionals and midwives, the vicious cycle of cesarean sections in Greece has not yet stopped.
\end{abstract}

Keywords: Cesarean section; High rates of cesarean section; Vicious cycle of cesarean deliveries; Cesarean section in Greece

\section{Introduction}

In recent years, we observed that Cesarean deliveries have increased and this is a global problem because of the negative impact on maternal and infant health (1). Although Cesarean Section (CS) is a very common surgery, it is associated with high rates of postoperative complications, such as hemorrhage, sepsis, ileus, thromboembolic events and uterine injury (2), (3), (4). Some other studies on neonatal child complications have shown that CS is associated with an increased risk for diabetes, obesity, atopic dermatitis and respiratory morbidity (5), (6). Furthermore, women having undergone a CS face an increased risk of placenta previa, especially if the second pregnancy occurs within a year after the CS (7).

In 1985, the World Health Organization [WHO] recommended that the ideal rates of CS range from 10\% to 15\%. The WHO also recommends that CS rates higher than these rates are not associated with a reduction in maternal-neonatal mortality (8). In 2009, the WHO recommended that the ideal CS rate was 5-15\% (9); however, at least 1.4 million cesarean sections were performed in Europe in 2017. In Greece, over half of all the deliveries, and in particular 58\% were CS performed (10). In 2016, the WHO conducted a survey on the high rates of CS in Greece and their results led to f recommendations to reduce unnecessary CS by training or retraining the medical personnel on natural birth and introducing strict control of medical indications for CSs in order to reach the WHO recognized rates (11). Apart from Greece, very high CS rates were in Romania 44.1\%, Cyprus 54.8\%, Bulgaria 43.1\%, Poland, 39.3\% and Hungary 37.3\% (12).

The reasons for this phenomenon and the extent of its consequences cannot be explained as a medical necessity. There are many CS associated factors ranging from medical and socio-economic to demographic and institutional some of which include purported medical as well as non-medical indications, such as maternal characteristics i.e. age and high

\footnotetext{
${ }^{*}$ Corresponding author: Evangelia Antoniou

Department of Midwifery, University of West Attica, Greece.
}

Copyright $(2021$ Author[s] retain the copyright of this article. This article is published under the terms of the Creative Commons Attribution Liscense 4.0. 
educational level, high-income level, and social class, maternal request, scientific-technological advances. Another important factor that contributes to the increase of CS rates is the Greek public health system, the costs of which are covered by the general government and the social health insurance system (13). Furthermore, there is an "under the table" payment system in Greece, especially in the field of obstetric services, as well as a clientele relationship between women and obstetricians to a great extent, which results in increased CS rates (14).

Another significant factor has been estimated to be a previous cesarean delivery. $66.1 \%$ of Greek women underwent an elective CS due to the previous CS (10). Therefore, it has been observed that a previous CS has become a major driver of high CS rates. So far, however, VBAC is not part of the culture of Greek obstetricians, due to their fear of uterine rupture from the first scar, although the vaginal birth after Cesarean [VBAC] is a major opportunity to stop this vicious cycle. In addition, a high percentage of obstetricians (15) prefer a medical health care, rather than a friendly and supportive health care based on midwifery practices (16). More specifically, the number of obstetricians in Greece was quite large in 1997, namely twice as many as in other European countries (13). Furthermore, although CSs are considered to be performed for the safety of mothers and infants, perinatal mortality rates in Greece are not lower than in countries with low percentages of CS rates (17), and this is contradictory because apparently CS does not fulfill the reason for being performed, but in most cases it is an unnecessary procedure.

Finally, instead of concluding, we would like to address all perinatal care professionals and researchers in this field, and raise the question about how we can we stop the vicious cycle of cesarean deliveries in Greece and how we will provide mothers with the opportunity to experience the childbirth they deserve.

\section{Conclusion}

To reduce CS rates, we need to focus on midwifery-obstetrician model of care, rather than an obstetrician model only. In addition, there is a great need of couple's psychoeducation during pregnancy and especially for pregnant women with specific mental health issues, such as fear of childbirth, anxiety or depression. Furthermore, the main goal should be to reduce the rates of primary CSs, since they are the starting point of a vicious cycle, with serious consequences for women's and infant health, psychology and society in general. This article aims to inform and raise awareness of obstetric and midwifery community about the need to make strategic decisions to reduce CS rates. Therefore, research on the effects of CSs and how to reduce them should be continued, especially in countries with high prevalence.

\section{Compliance with ethical standards}

\section{Acknowledgments}

We would like to thank the members of the research laboratory "The Laboratory of Midwifery Care during Antenatal and Post natal period - Breastfeeding" of the University of West Attica, for their valuable help in writing this article and for the continuous support of our research.

\section{Disclosure of conflict of interest}

There are no conflicts of interest.

\section{References}

[1] Ayres-De-Campos D, Cruz J, Medeiros-Borges C, Costa-Santos C, Vicente L. Lowered national cesarean section rates after a concerted action. ActaObstetGynecolScand [Internet]. 2015 [cited 2020 Apr 6]; 94 [4]: 391-8.

[2] Field A, Haloob R. Complications of caesarean section. ObstetGynaecol [Internet]. 2016 [cited 2020 Apr 7]; 18 [4]: 265-72.

[3] Walker I. Thrombophilia in pregnancy. J ClinPathol [Internet]. 2000 Aug [cited 2020 Jun 20]; 53[8]: 573-80.

[4] Blondon M, Casini A, Hoppe KK, Boehlen F, Righini M, Smith NL. Risks of Venous Thromboembolism After Cesarean Sections: A Meta-Analysis. Chest. 2016 Sep; 150[3]: 572-96.

[5] Cardwell CR, Stene LC, Joner G, Cinek O, Svensson J, Goldacre MJ, et al. Caesarean section is associated with an increased risk of childhood-onset type 1 diabetes mellitus: a meta-analysis of observational studies. Diabetologia. 2008 May; 51[5]: 726-35. 
[6] Magnus MC, Håberg SE, Stigum H, Nafstad P, London SJ, Vangen S, et al. Delivery by Cesarean section and early childhood respiratory symptoms and disorders: the Norwegian mother and child cohort study. Am J Epidemiol. 2011 Dec 1; 174[11]: 1275-85.

[7] Getahun D, Oyelese Y, Salihu HM, Ananth CV. Previous cesarean delivery and risks of placenta previa and placental abruption. Obstet Gynecol. 2006 Apr; 107[4]: 771-8.

[8] Betran A, Torloni M, Zhang J, Gülmezoglu A, Aleem H, Althabe F, et al. WHO Statement on Caesarean Section Rates. Bjog [Internet]. 2016 Apr [cited 2020 Mar 23]; 123[5]: 667-70.

[9] Bailey P, Lobis S, Fortney J, Maine D. Family Health International [Organization], Joseph L. Mailman School of Public Health, et al., editors. Monitoring emergency obstetric care: a handbook. Geneva, Switzerland: World Health Organization. 2009; 152.

[10] Antoniou E, Orovou E, Iliadou M, Sarella A, Palaska E, Sarantaki A, et al. Factors Associated with the Type of Cesarean Section in Greece and Their Correlation with International Guidelines. Acta Inform Medica [Internet]. 2021 Mar [cited 2021 May 29]; 29[1]: 38-44.

[11] Greece commits to addressing excessive reliance on caesarean sections [Internet]. [cited 2020 Oct 18].

[12] Large differences in share of caesarean births [Internet]. [cited 2020 Apr 8].

[13] Mossialos E, Allin S, Karras K, Davaki K. An Investigation of Caesarean Sections in Three Greek Hospitals: The Impact of Financial Incentives and Convenience. Eur J Public Health. 1 Jul 2005 ; 15: 288-95.

[14] KaitelidouDCh, Tsirona CS, Galanis PA, SiskouOCh, Mladovsky P, Kouli EG, et al. Informal payments for maternity health services in public hospitals in Greece. Health Policy [Internet]. 2013 Jan; 109[1]: 23-30.

[15] Alran S, Sibony O, Oury J-F, Luton D, Blot P. Differences in management and results in term-delivery in nine European referral hospitals: descriptive study. Eur J ObstetGynecolReprod Biol. 2002 Jun 10; 103 [1]: 4-13.

[16] Wang Z, Sun W, Zhou H. Midwife-led care model for reducing caesarean rate: A novel concept for worldwide birth units where standard obstetric care still dominates. J Med Hypotheses Ideas [Internet]. 2012 Jan 1 [cited 2020 Apr 12]; 6[1]: 28-31.

[17] European Perinatal Health Report 2015 - Euro-Peristat [Internet]. [cited 2020 Sep 18]. 